\title{
Is the antimicrobial removal device a cost-effective addition to conventional blood cultures?
}

\author{
ROSEMARY MUNRO, PETER J COLLIGNON, TANIA C SORRELL, PETER TOMLINSON
}

From the Bacteriology Department and Infectious Disease Medical Unit, Westmead Centre, Westmead, New South Wales, Australia

SUMMARY Two hundred and thirty-four blood cultures from 140 patients receiving antibiotics were processed using the antimicrobial removal device (ARD) in parallel with conventional blood cultures. One hundred and seventy cultures were obtained from patients suspected to have bacteraemia and 64 from patients known to have a positive conventional blood culture within the preceding three days.

A total of $38(16.2 \%)$ ARD-processed cultures were positive, compared with $21(8.9 \%)$ conventional cultures $(p<0.0001$, Fisher's exact test). No instances of positive conventional cultures and negative ARD-processed cultures were identified. Thirty-three of 38 ARDprocessed cultures became positive within $24 \mathrm{~h}$, compared with 14 parallel conventional cultures $(p<0.0001$ Fisher's exact test). Although the yield and rapidity of isolation of bacteria from blood were improved by ARD processing, in only one of 140 patients did this information alter treatment. As the use of an ARD is associated with a sixfold increase in the cost of blood cultures, we conclude that, in our hands, general use of the device in patients receiving antibiotics is not cost-effective. Considerable care should be taken in selecting patients for ARD-processing of blood cultures.

The antimicrobial removal device or ARD (Marion Scientific, Missouri, USA) contains a mixture of cationic and polymeric adsorbent resins in saline with sodium polyethanolsulphonate. The resins have been pretreated to prevent bacterial retention and to remove up to $100 \mathrm{mg} / \mathrm{l}$ of antibiotic and other bacterial inhibitors from the blood. The ARD was developed by Melnick and Wallis, who claimed that its use in bacteraemic patients receiving antibiotics permitted earlier isolation of the causative organism. In some cases, blood cultures were only positive after processing with the ARD.'

The diagnosis of bacteraemia in a patient who is receiving antibiotics may be difficult as antibiotics present in the blood are inoculated into the culture medium and may inhibit bacterial growth or prolong the time required for the culture to become positive.

This study was undertaken to assess whether an ARD was a useful and cost-effective addition to standard blood cultures in patients suspected to be bacteraemic who were receiving antibiotics.

Accepted for publication 30 November 1983

\section{Material and methods}

The study was conducted at the Westmead Centre, Sydney, between April 1982 and February 1983.

\section{PATIENTS}

Two groups of patients were included in this study. Eighty-three patients in group $\mathrm{A}$ were receiving empirical antibiotic therapy for suspected bacteraemia. Patients in group B were receiving antibiotics for bacteraemia confirmed by conventional blood culture within the preceding three days. Thirty-one patients in group A were located in the Intensive Care Unit. The diagnosis of suspected bacteraemia was made by the Intensive Care physicians. Fifty-two remaining patients in group A were assessed clinically as bacteraemic by the Infectious Diseases Unit. Minimal criteria for diagnosis in immunosuppressed neutropaenic patients included an unexplained temperature of $\geqslant 38^{\circ} \mathrm{C}$ on two occasions more than four hours apart. Bacteraemia was suspected in other patients in the presence of a temperature greater than $38^{\circ} \mathrm{C}$, rigors and signs of systemic toxicity. 
COLLECTION AND PROCESSING OF SPECIMENS

Patients in group A had one blood culture, or two blood cultures taken at least one hour apart. Blood cultures were taken from patients in group $B$ on one occasion.

After preparation of the skin with alcohol, $10 \mathrm{ml}$ of blood was withdrawn and aseptically dispensed, $5 \mathrm{ml}$ into the ARD bottle and $5 \mathrm{ml}$ into the conventional supplemented peptone broth II culture (Becton-Dickinson, New Jersey, USA). Only blood cultures taken between the hours of $0800 \mathrm{~h}$ and $1600 \mathrm{~h}$ were included in the study as reduced staffing of the laboratory outside these hours precluded the processing of ARD blood cultures.

The conventional and ARD blood cultures were returned to the laboratory within two hours of collection and the ARD bottle placed on a rotator (Marion Scientific) for $15 \mathrm{~min}$. The blood saline mixture was aspirated aseptically from the ARD bottle with a needle and syringe and transferred to a second blood culture bottle containing supplemented peptone broth. Both the conventional blood culture and ARD-processed blood cultures were incubated at $35^{\circ} \mathrm{C}$ and subcultured similarly. Subculture was performed after three hours' incubation if this were possible within the normal working day and after $24 \mathrm{~h}, 48 \mathrm{~h}, 7$ days, and 14 days of incubation, or when growth was detected macroscopically. Broths were subcultured on to chocolate agar, MacConkey agar, and enriched blood agar. Chocolate agar plates were incubated for $48 \mathrm{~h}$ at $35^{\circ} \mathrm{C}$ in $7 \% \mathrm{CO}_{2}$, MacConkey agar plates for $24 \mathrm{~h}$ at $35^{\circ} \mathrm{C}$ aerobically, and enriched blood agar plates for $48 \mathrm{~h}$ at $35^{\circ} \mathrm{C}$ anaerobically.

\section{COST OF ARD PROCESSING}

The cost of an antimicrobial removal device (ARD) is approximately five times the cost of a supplemented peptone broth blood culture. In addition, technical time is required in the initial processing of the ARD culture. This involves shaking of the ARD bottle on a rotator for $15 \mathrm{~min}$ and aseptic dispensing of the blood saline mixture into a blood culture bottle. We estimate that processing of a blood culture using an ARD increases the cost of the blood culture by a factor of six.

\section{CLINICAL DATA}

Details of antibiotic treatment were recorded. Patients with blood cultures confirmed to be positive by conventional methods in the preceding three days and patients whose blood cultures subsequently became positive during the study were followed up daily by the Infectious Diseases Unit. The initial antibiotic therapy received by these patients was assessed by the Infectious Diseases Unit as being: (a) Optimal (organism isolated sensitive to at least one antibiotic used, dose judged to be adequate and supported by serum assays in the case of aminoglycosides).

(b) Suboptimal (organism sensitive to at least one antibiotic used but the antibiotic not the agent of choice for that organism or antibiotic used in inadequate dosage).

(c) Unsuitable (organisms resistant to all antibiotics used).

Patients were assessed by their response to therapy, development of complications, and ultimate outcome. Alterations in management which occurred as a result of the positive ARD culture were recorded.

\section{Results}

One hundred and seventy parallel cultures were obtained from 83 patients in group A. Sixty-four separate episodes of bacteraemia were identified in 57 patients in group B. Antibiotic therapy administered at the time blood cultures were taken included at least one of the following agents:

penicillin, ampicillin, cloxacillin, flucloxacillin, cephalexin, cephalothin, cephamandole, cefoxitin, cefotaxime, cotrimoxazole, rifampicin, fusidic acid, vancomycin, gentamicin, tobramycin, and amikacin.

Thirty-eight of the 234 ARD-processed blood cultures $(16.2 \%)$ were positive, $12(31.5 \%)$ from patients in group $\mathrm{A}$ and $26(68.5 \%)$ from patients in group B. Bacteria isolated are listed in Table 1. Yields from ARD-processed and conventional blood cultures are summarised in Table 2. As shown in Table 3, bacteria were isolated earlier from ARD-processed cultures than from conventional cultures.

Antibiotic therapy was classified as unsuitable in three of the patients with positive ARD cultures;

Table 1 Bacteria isolated from blood cultures

\begin{tabular}{|c|c|c|c|}
\hline \multirow[t]{2}{*}{ Organism } & \multicolumn{3}{|c|}{ No of isolates } \\
\hline & Group $A$ & Group B & Total \\
\hline $\begin{array}{l}\text { Gram-positive } \\
\text { Staphylococcus aureus } \\
\text { Staphylococcus epidermidis } \\
\text { Streptococcus faecalis }\end{array}$ & $\begin{array}{l}5 \\
1 \\
2\end{array}$ & $\begin{array}{r}16 \\
0 \\
0\end{array}$ & $\begin{array}{r}21 \\
1 \\
2\end{array}$ \\
\hline $\begin{array}{l}\text { Gram-negative } \\
\text { Escherichia coli } \\
\text { Acinetobacter calcoaceticus } \\
\text { Klebsiella oxytoca } \\
\text { Proteus mirabilis } \\
\text { Enterobacter cloacae } \\
\text { Pseudomonas aeruginosa }\end{array}$ & $\begin{array}{l}3 \\
0 \\
0 \\
0 \\
0 \\
1\end{array}$ & $\begin{array}{l}6 \\
1 \\
1 \\
1 \\
1 \\
0\end{array}$ & $\begin{array}{l}9 \\
1 \\
1 \\
1 \\
1 \\
1\end{array}$ \\
\hline & 12 & 26 & 38 \\
\hline
\end{tabular}


Table 2 Yield of positive ARD and conventional blood cultures from patients receiving antibiotics

\begin{tabular}{llllll}
\hline Conventional & \multicolumn{2}{l}{ Group $A(n=170)$} & & Group $B(n=64)$ \\
\cline { 2 - 3 } \cline { 5 - 6 } & $A R D(+)$ & $A R D(-)$ & & $A R D(+)$ & $A R D(-)$ \\
\hline Positive & 7 & 0 & & 14 & 0 \\
Negative & 5 & 158 & & 12 & 38 \\
\hline
\end{tabular}

$\mathbf{n}=$ number of parallel blood cultures.

In both patient groups, significantly more positive results were obtained by processing with the ARD ( $p<0.0001$ in each case, Fisher's exact test)

conventional cultures were also positive in these patients. All other positive ARD-processed cultures were obtained from patients receiving at least one antibiotic to which the organism was sensitive on in vitro testing. In four of these cases the antibiotic choice was assessed as suboptimal (for example, the use of tobramycin alone for the treatment of bacteraemia due to Pseudomonas aeruginosa). The parallel conventional blood culture was positive in each of these four cases. Clinical management was altered by the identification of a positive ARDprocessed culture in only one case, a patient in group A. This 20-year-old man had received a bone marrow transplant for acute leukaemia. He developed bacteraemia and meningitis due to methicillin-resistant Staphylococcus aureus. During the course of therapy with vancomycin and fusidic acid, methicillin-fusidic acid-resistant Staphylococcus aureus was isolated from an ARD-processed blood culture. The parallel conventional culture remained negative. Rifampicin was substituted for fusidic acid, and the patient recovered.

\section{Discussion}

The use of an ARD in patients receiving antibiotics resulted in an increased yield of positive blood cultures, and cultures becoming positive earlier than with a conventional system. There were no organisms isolated from conventional cultures that were

Table 3 Duration of incubation before culture positivity in 38 paired $A R D$ and conventional blood cultures

\begin{tabular}{lcc}
\hline Time & ARD blood culture & $\begin{array}{c}\text { Conventional } \\
\text { blood culture }\end{array}$ \\
\hline $3 \mathrm{~h}$ & $0^{*}$ & $0^{*}$ \\
$24 \mathrm{~h} \dagger$ & 33 & 14 \\
$48 \mathrm{~h}$ & 5 & 4 \\
7 days & 0 & 1 \\
$10-14$ days & 0 & 2 \\
Negative at 14 days & 0 & 17 \\
\hline
\end{tabular}

*Only 16 cultures were sampled after three hours' incubation. †Compared with conventional cultures, significantly more ARD cultures were positive within $24 \mathrm{~h}$ of incubation ( $\mathrm{p}<0.0001$, Fisher's exact test). not also isolated from ARD cultures. In 17 of 38 cases, cultures were only positive when processed with an ARD. Despite this information obtained from using the ARD, antimicrobial therapy was changed in only one of 83 patients with suspected bacteraemia.

Reports of the efficacy of the ARD have varied in different centres. Wallis et $a l^{1}$ performed parallel ARD and conventional blood cultures on 51 patients receiving antibiotics for suspected septicaemia. Blood cultures were positive in 31 cases. In 21 of these, organisms were isolated more rapidly after processing with an ARD. Four patients had blood cultures which were positive only after use of the ARD. Appleman et $a l^{2}$ studied 300 patients diagnosed as bacteraemic despite antibiotic therapy-of 53 patients with positive blood cultures, 20 had positive cultures only from ARD-processed bottles and seven had positive cultures in specimens processed in the conventional manner, with negative ARD-processed bottles. Cultures became positive earlier with ARD-processed specimens.

Conventional and ARD-treated blood cultures have also been compared in patients suspected to be bacteraemic, whether or not they were receiving antibiotics. Wright $e t a^{3}$ demonstrated no advantages of ARD processing over conventional cultures in 87 bacteraemic patients, 28 of whom were receiving antibiotics. Of these 28 patients the ARD yielded the only positive result in three cases. The authors suggested that the inclusion of sodium polyethanolsulphonate in their conventional culture media may have improved bacterial yields and rapidity of isolation. Strand ${ }^{4}$ concluded that there was no advantage to using the ARD in conjunction with a three-bottle BACTEC (Johnson Laboratories, USA) system in clinically bacteraemic patients.

In our study, of 170 cultures from 83 patients receiving antibiotics for suspected bacteraemia, only $12(7 \cdot 1 \%)$ were positive when processed by ARD; seven were also positive using conventional cultures. This is a lower yield than that reported by Wallis $e t$ $a l^{1}(62 \%)$ or Appleman et al ${ }^{2}(18 \%)$. Both of these studies compared single, parallel, conventional and ARD cultures in patients suspected to be bacteraemic while receiving or having recently received antibiotic therapy. The lower yield in our series may reflect the difficulty in making a clinical diagnosis of bacteraemia in seriously ill patients in Intensive Care and Haematology Units.

Twenty-six of the 38 positive cultures obtained were from 57 patients with a conventional blood culture positive within the preceding three days. The clinical significance of these positive cultures which occurred despite appropriate antibiotic therapy is 
difficult to assess. Washington, ${ }^{5}$ recorded persistent Staphylococcus aureus bacteraemia for at least $48 \mathrm{~h}$ in 23 of 25 ARD-processed blood cultures from patients receiving antibiotics. Our study demonstrated persistent bacteraemia not only with Staphylococcus aureus, but with a range of Gramnegative organisms.

In the course of this study, 300 antimicrobial removal devices (ARD) were supplied to the Intensive Care Unit and the Infectious Disease Unit. Two hundred and thirty-four ARD cultures were processed-66 ARDs were wasted due to being collected inadvertently outside laboratory working hours, or taking in excess of two hours to reach the laboratory.

We conclude that general use of the ARD, which at our hospital increases the cost of each blood culture by a factor of 6 , is not cost-effective. That improved treatment followed use of the ARD in a patient responding poorly to antibiotics, suggests the device is of value in selected cases.

We wish to thank Swift Watts Winter Company,
Sydney, for supplying the antimicrobial removal devices for this study, and Miss S Summerill for her valuable secretarial assistance.

\section{References}

' Wallis C, Melnick JL, Wende RD, Riely PE. Rapid isolation of bacteria from septicaemic patients by use of an antimicrobial agent removal device. J Clin Microbiol 1980;11:462-4.

2 Appleman MD, Swinney RS, Heseltine PNR. Evaluation of the antibiotic removal device. J Clin Microbiol 1982;15:278-81.

${ }^{3}$ Wright AJ, Thompson RL, McLimans CA, Wilson WR, Washington JA. The antimicrobial removal device. Am J Clin Pathol 1982;78:173-7.

${ }^{4}$ Strand CL. Evaluation of the antimicrobial removal device when used with the BACTEC blood culture system. Am J Clin Pathol 1982;78:853-6.

5 Washington JA. The role of the microbiology laboratory in the diagnosis and antimicrobial treatment of infective endocarditis. Mayo Clin Proc 1982;57:22-32.

Requests for reprints to: $\operatorname{Dr} \mathrm{R}$ Munro, Bacteriology Department, Institute of Clinical Pathology and Medical Research, Westmead Centre, Westmead, NSW 2145, Australia. 